\title{
AN EXTRA LARGE CHOANAL POLYPS
}

\author{
Nedev $\mathbf{P}$. \\ Department of Neurosurgery, Othorhinolaryngology and Ophthalmology - Medical University \\ Varna; Clinic of Otorhinolaringology - University Hospital "Saint Marina" - Varna
}

Reviewed by: Assoc. Prof. R. Radev, PhD

\begin{abstract}
Nasal polyps are caused by a multitude of factors. The most common causes are repeated bouts of sinusitis, allergy, chronic rhinitis, infectious rhinosinusitis, and asthma. In this paper we present two cases of extra large choanal polyps - in adolescent and an adult. The polyps were extracted successfully and the patients remained symptom-free at the 1-year follow-up period. We discuss the management of the nasal polyps of extra size.
\end{abstract}

Keywords: nasal polyps, allergic rhinitis, asthma, choanal polyps

\section{INTRODUCTION}

Nasal polyps consist of oedematous mucous membrane infiltrated by lymphocytes, plasma cells and large numbers of eosinophils. (1) They result from prolapse of the mucosal lining of the nose and sinuses, in particular from the lining of the ethmoid and maxillary sinuses, protruding down into the middle meatus and presenting as smooth, round, pale and translucent swellings. (2)

The aetiology of the nasal polyps is unknown. Allergy is a suspected factor, but this is still controversial. (3) Vasomotor imbalance, exposure to irritant substances and infections are all possible factors for nasal polyp aetiology. (4) Usually nasal polyps are accompanied with a chronic rhinosinusitis. It is debated whether chronic rhinosinusitis with and without polyps represent different stages of one single disease or two different disease entities. The presence of nasal polyposis in asthma patients is associated with an increase in asthma severity.

\section{CASE REPORTS}

\section{Case 1.}

A 14-year-old boy presented with complaints of increasing difficulty in nasal breathing and difficulty swallowing solid food. His symptoms had been present for approximately 1 year, and they were associated with a feeling of obstruction in the throat. According to his parents, the patient snored loudly during the night and present all signs of sleep apnea syndrome. These symptoms were accompanied with frequent otitis, hearing decrease and smell lost. The patient reported incidences of allergic rhinitis. The patient's nasal function was checked on the basis of the following criteria: nasal obstruction, rhinorrhea, hearing, facial pain, and the loss of sense of smell. The endonasal microscope examination revealed small polyps mass in meatus nasi inferior end.

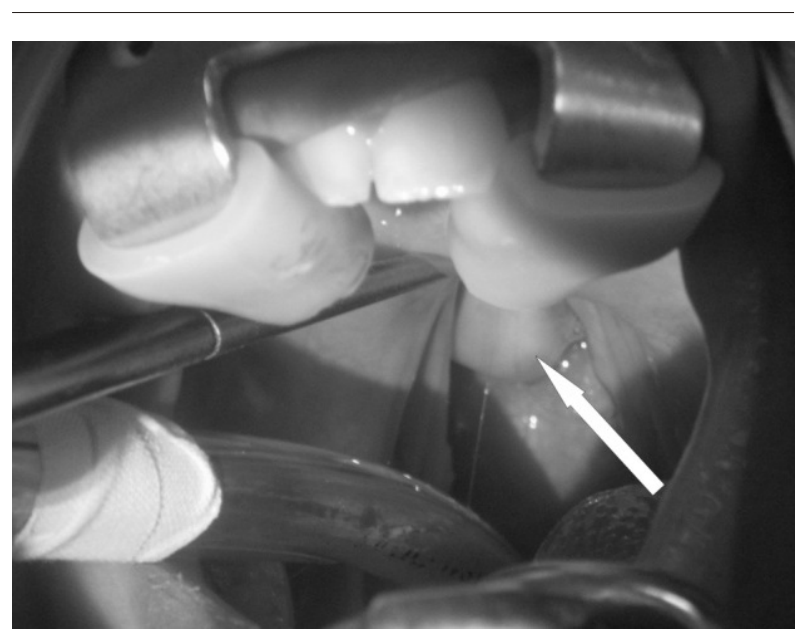

Figure 1 Intraoral examination - view of a large and roundish soft-tissue mass in the oropharynx - case 1.

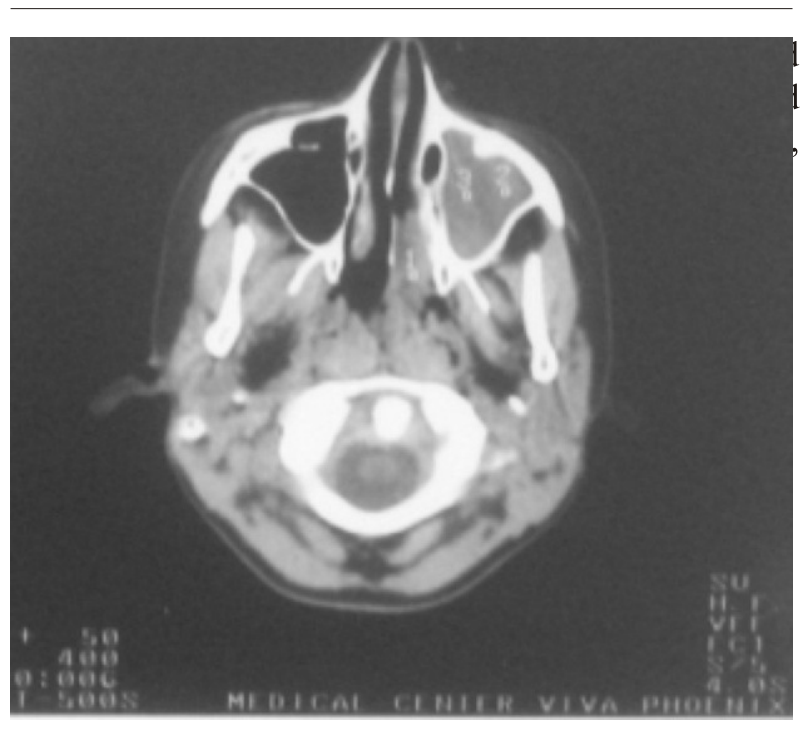

Figure 2 Computer tomography of the nasal sinuses case 1 . 
protrude through the choana into the nasopharynx. (Figure 2)

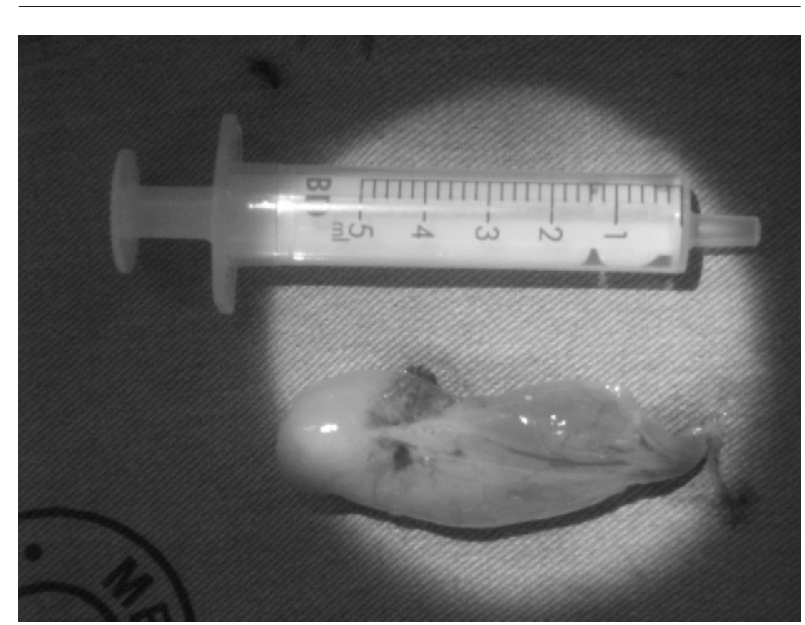

Figure 3 Choanal polyps after extraction - macroscopic view - case 1.

The patient underwent transoral extaction of the mass under general anesthesia. The choanal polyps mass was extracted in a whole. (Figure 3) The size of the polyp mass was about $6.3 \mathrm{sm}$. The histopathologic diagnosis was a benign polyp. The hyposensibilisation therapy was realized postoperative for about 3 months. One year after the surgery there were no recurrences. The patient noted significant improvement in breathing and swallowing. The nasal function was completely rehabilitated and all of the above symptoms were disappeared.

\section{Case 2.}

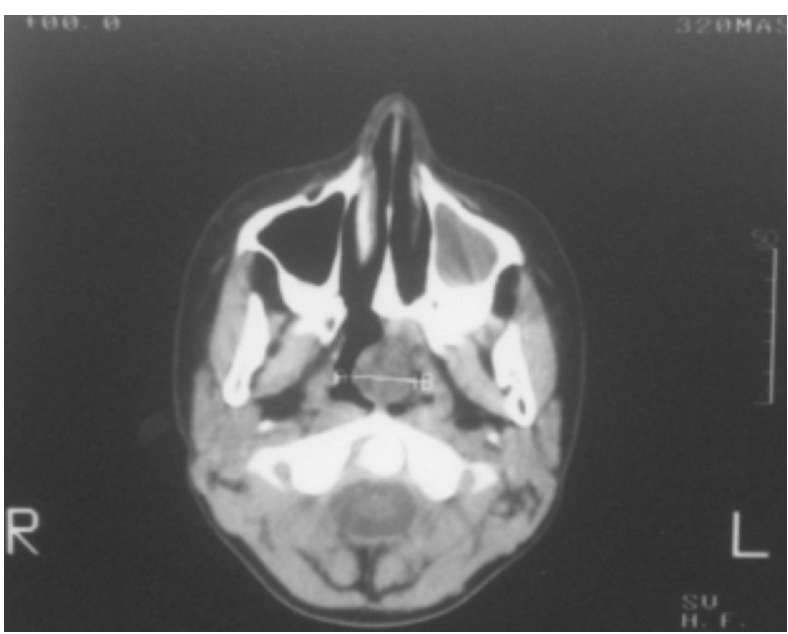

Figure 4 Computer tomography of the paranasal sinuses - case 2.

A 36-year-old man presented with a 2-year history of bilateral nasal obstruction, worsening snoring, daytime somnolence, and altered voice (rhinolalia clausa). The anamnesis shows sleep apnea symptoms. He was forced to sleep sitting up because the upper airway obstruction would not allow him to breathe when he was lying down. He reported that he usually had treated the nasal obstruction with nasal drops, but they were ineffective. In his childhood he was suffering from spastic bronchitis and incidences of allergic rhinitis.

On examination, a huge choanal polyp could be seen passing from the nasopharynx down into the oropharynx. CT of the paranasal sinuses revealed a large perfectly round soft-tissue opacity in the nasopharynx (Figure 4) that had its origin in the maxillary sinus. CT also demonstrated a complete opacification of the maxillary sinuses. The sphenoid sinus and ethmoidal cells were clear.

The patient underwent transoral extaction of the mass under local anesthesia with $10 \%$ lidocaine spay. The choanal polyp was removed in one step extraction. The choanal polyp size was over 10 sm with the pedicule. (Figure 5) The histopathologic diagnosis was a benign polyp. We applied a local therapy with Flixonase Allergy Nasal Spray for one month period. One year postoperatively the nasal function was completely rehabilitated but the allergic symptoms still existed. The night sleep was improved. The presented rhinolalia clausa was disappeared. The patient has not reported recurrences.

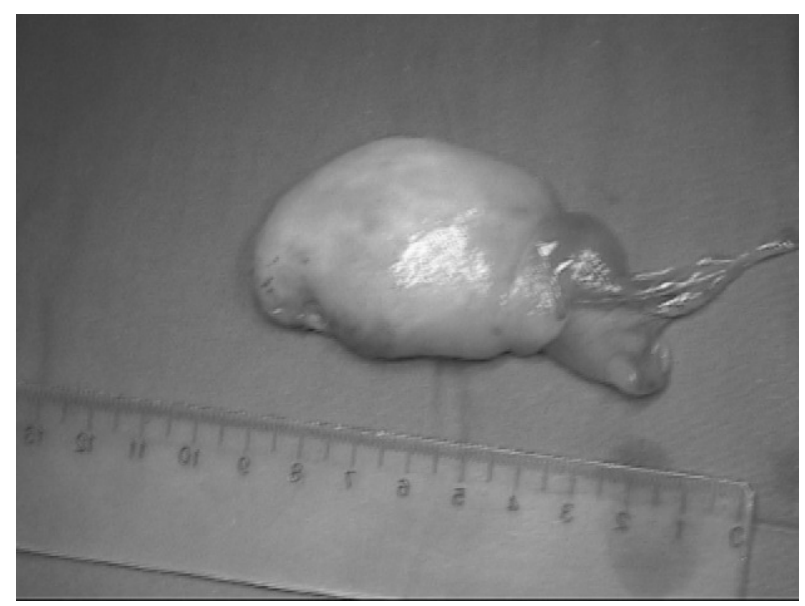

Figure 5 Choanal polyps after extraction - macroscopic view - case 2 .

\section{DISCUSSION}

Choanal polyps are benign masses that protrude through the choana into the nasopharynx. They are classified as antrochoanal and sphenochoanal, depending on the sinus of origin. Antrochoanal polyps arise from the maxillary sinus and are more common - they account for 3 to $6 \%$ of all polyps. (5) Most sphenochoanal polyps are not associated with other sinus disease although in some cases, other sinuses are affected, possibly as a result of blockage by the polyp. (5)

Nasal polyposis is one of the most common inflammatory pathologies of the nasal cavity and paranasal sinuses, and its prevalence is around $2.7 \%$ in Europe (6). On the other hand, allergic rhinitis is a high prevalence disease in developed countries, affecting about $10-20 \%$ of the general pop- 
ulation. (7)A variety of symptoms attached to these two conditions (i.e. nasal polyposis and allergic rhinitis) testify to the serious alteration of the nasosinusal function: airway blockage, anterior and posterior rhinorrhea, stuffiness and facial pain, sneezing episodes, loss of the sense of smell, alteration of the sense of flavor.

Many patients with nasal polyposis have positive allergic tests. Nevertheless, the relationship between nasal polyposis and allergy is not clear in the literature. The presence or severity of nasal symptoms in patients with nasal polyposis is correlated with the presence of positive allergic tests. (8) In general, the nasal polyps in patients with asthma tend to be more severe and large than they are in patients without asthma. (9)

Most choanal polyps originate in the maxillary sinus and extend into the nasopharynx through the natural or accessory ostium in the middle meatus, or through a postsurgical middle or inferior meatal antrostomy. CT usually shows choanal polyp in association with ipsilateral maxillary sinusitis. Rarely do choanal polyps arise from the sphenoid or ethmoid sinus or their ostia. Some choanal polyps extend down into the oropharynx. In the two cases described herein, the origin of the mass was identified preoperatively. Ordinarily, the oropharyngeal area can be easily visualized by transnasal or transoral endoscopy but we recommend microscopic examination. In the two presented cases the mass of the polyps was extra large; therefore the extraction was easily performed, completely removed with the pedicule.

The differential diagnosis of large nasopharyngeal masses should include benign disease such as juvenile angiofibroma, teratoma, meningoencephalocele, chordoma, paraganglioma, nasopharyngeal extension of a parapharyngeal parotid tumor and malignant disease such as carcinoma, lymphoma or sarcoma. A thorough endoscopic examination of the nose and nasopharynx is important to make a diagnosis of a nasopharyngeal lesion. (10) Our experience with endonasal microscope examination shows excellent visualization of all cases of nasal polyposis. CT and/or magnetic resonance imaging can determine the localization, size, and extent of a nasopharyngeal mass. For a vascular lesion, magnetic resonance angiography of the nasopharynx is needed. These methods are decisive in differential diagnosis terms. Several studies have reported that patients with asthma, compared with those without, have more severe sinus disease and nasal polyposis. In our study, because the two patients reported allergic symptoms, we recommend further regular preventive examinations. Then the consultation with the ENT specialist should be conducted with specialist of clinical immunology and allergology.

\section{CONCLUSIONS}

Preoperative identification of the sinus from which a choanal polyp arises is important because the polyp's site of origin in the sinus must be managed in order to minimize recurrence. In patients with asthma, the possibility of polyps should be investigated and treatment planned accordingly. To manage a large nasopharyngeal polyp such as these two cases, we recommend an transoral extraction of the total mass, packing and postoperatively surveillance of the local general condition. The cooperation of ENT specialist, pneumonologist and allergologist is needed.

\section{REFERENCES}

1. Mygind N, Dahl R, Bachert C. Nasal polyposis, eosinophil dominated inflammation, and allergy. Tho$\operatorname{rax} 2000$; 55: S79-S83.

2. Durham SR, Mackay LS. Diseases of the nose. In: Gibson GJ, Geddes DM, Costabel U, Sterk PJ, Corrin B (eds). Respiratory Medicine, 3rd edn. Saunders, London, 2003; 1035-47.

3. Ceylan E., Gencer M., San I. Nasal polyps and the severity of asthma Respirology (2007) 12, 272-276

4. Drake-Lee AB. Nasal polyps. In: Kerr AG (ed.). Rhinology, Vol. 4. Scott-Brown's Otolaryngology, London, 1997; 1-15.

5. Tysome, James R., Saleh, Hesham A. Sphenochoanal polyp presenting with concomitant nasal polyps., Ear, Nose \& Throat Journal, 2007, Vol. 86/1

6. Johansson L, Akerlund A, Holmberg K, Melen I, Bende M. Prevalence of nasal polyps in adults: the Skovde populationbased study. Ann Otol Rhinol Laryngol 2003;/112:/625-629.

7. Van Cauwenberge P, Bachert C, Passalacqua G, Bousquet J, Canonica GW, Durham SR, et al. Consensus statement on the treatment of allergic rhinitis. Allergy 2000;/55:/116-134.

8. Bonfils P. Avan P. Malinvaud D. Influence of allergy on the symptoms and treatment of nasal polyposis Acta Oto-Laryngologica, 2006; 126: 839-844

9. Larsen K. The clinical relationship of nasal polyps to asthma. Allergy Asthma Proc 1996; 17(5):243-9.

10. Chen E., Yanagisawa E. An unusually large choanal polyp that almost completely obstructed the oropharyngeal airway Ear, Nose \& Throat Journal $2006474-476$ 